\title{
8 Glaube und Religion
}

Dieses Kapitel wurde bei Erstveröffentlichung ohne die korrekte Creative Commons Lizenz veröffentlicht. Die korrekte Lizenz finden Sie am Ende des Kapitels.

Ein Erratum zu diesem Kapitel ist verfügbar unter DOI 10.1007/978-3-658-12533-2_14

(C) SINUS Markt- und Sozialforschung GmbH 2016

M. Calmbach et al., Wie ticken Jugendliche 2016?, DOI 10.1007/978-3-658-12533-2_8 
Wie in vielen anderen Lebensbereichen hat auch im Hinblick auf Glaube und Religion in den letzten Jahrzehnten eine Pluralisierung stattgefunden. Das Spektrum religiöser Gemeinschaften hat sich innerhalb des Christentums (z. B. Freikirchen, charismatische und evangelikale Gruppierungen), aber auch durch die Verbreitung nichtchristlicher Religionen, v. a. des Islam, in Europa enorm erweitert. Hinzu kommen nicht unmittelbar religionsgebundene Formen der Spiritualität.

Schon die SINUS-Jugendstudien 2008 und 2012 haben zum Themenkomplex Glaube, Religion und Kirche gezeigt, dass Jugendliche ein Bedürfnis nach Sinnfindung haben, dieses jedoch häufig als "religiöse Touristen" mit einem individuell zusammengestellten Patchwork aus einer Vielzahl von religiösen, quasireligiösen bzw. spirituellen Angeboten befriedigen. Sie finden den gesuchten Sinn also heute nicht mehr zwingend in einer Religion oder Kirche, sondern sie entwickeln aus verschiedenen Quellen einen „persönlichen Glauben“. Dieser Glaube ist für Jugendliche veränderbar und individuell, während Religion und Kirche eher als institutionell und damit unbeweglich wahrgenommen werden. Trotzdem gehört nach wie vor die Mehrheit junger Menschen in Deutschland einer Glaubensgemeinschaft oder Kirche an. Konfessionslos sind laut der aktuellen Shell-Jugendstudie nur 23\% der 12- bis 25-Jährigen in Deutschland.

Seit Erscheinen der letzten SINUS-Jugendstudie gab es zahlreiche Ereignisse rund um Glaube, Religion und Kirche, die von den Medien und einer breiten Öffentlichkeit aufmerksam verfolgt wurden. Man denke hier nur an den historisch einmaligen Rücktritt von Papst Benedikt, dem ersten Papst deutscher Herkunft seit vielen Jahrhunderten, und die Wahl von Papst Franziskus im Jahr 2013. Schon kurze Zeit später löste der Veruntreuungsskandal um den damaligen Limburger Bischof Franz-Peter Tebartz-van Elst eine breite Diskussion über die Strukturen und Vermögensverhältnisse der katholischen Kirche aus. Für Jugendliche, die sich in der evangelischen Kirche engagieren, sind die ersten Vorzeichen des Lutherjahres 2017 erkennbar, mit dem 500 Jahren Reformation in Deutschland gedacht wird.

Vor allem durch die Terroranschläge von Al Qaida und spätestens seit 2013 durch die kriegerischen Aktivitäten des sogenannten "Islamischen 
Staates" in Syrien und dem Irak ist die Diskussion über den Islam und verschiedene Formen des Islamismus wiederbelebt worden. In Dresden gründete sich 2014 die rechtspopulistische Bewegung "Patriotische Europäer gegen die Islamisierung des Abendlandes" (Pegida), in der sich sehr deutlich islamophobe und fremdenfeindliche Tendenzen zeigen. Die Terroranschläge in Frankreich im Januar und November 2015 markieren einen neuen negativen Höhepunkt von Gewalt, die vordergründig im Namen einer Religion verübt wird und erhebliche Konsequenzen für den Umgang mit religiöser Vielfalt hat. Besondere Relevanz hat diese Entwicklung mit Blick auf die wachsende Zahl Geflüchteter verschiedener nationaler, kultureller und religiöser Herkunft in Deutschland und die damit verbundene große Herausforderung der kommenden Jahre, einen erfolgreichen Umgang mit dieser v. a. religiösen Vielfalt zu bewerkstelligen.

Vor dem Hintergrund dieser Ereignisse war ein Auftrag der aktuellen Studie, erneut nach Glaube und Religion im Alltag junger Menschen zu fragen. Dabei richtet sich der Blick zunächst auf die eigene Zugehörigkeit zu Glaubensgemeinschaften und die Frage, wie stark die Bindung in diesem Bereich ist und worauf sie sich stützt. Ebenso wurden die Jugendlichen dazu befragt, ob und wie sich Glaube und Religion in ihrem konkreten Alltag äußern und was es für sie bedeutet, den eigenen Glauben zu leben. Dabei werden im Folgenden auch Unterschiede zwischen christlichen und muslimischen Jugendlichen beleuchtet.

Aufgrund der beschriebenen aktuellen politischen und gesellschaftlichen Entwicklungen liegt bei der diesjährigen Studie ein besonderer Fokus auf dem Umgang der Jugendlichen mit religiöser Vielfalt. Gefragt wurde nach der religiösen Vielfalt im persönlichen Umfeld (v. a. im Freundeskreis), dem alltäglichen Austausch über Religion sowie nach konkreten Berührungspunkten mit anderen Religionen. Ein weiteres Interesse galt der Frage, ob und wie Jugendliche religiöse Konflikte wahrnehmen, wie sie diese bewerten und inwiefern diese einen Einfluss auf ihren persönlichen Glauben sowie ihre grundsätzliche Einstellung zu Glaube und Religion haben. 


\title{
8.1 Zugehörigkeit zu einer Glaubensgemeinschaft
}

\author{
Die individuelle Auffassung von Glaube und Religion ist \\ nicht nur durch die Lebenswelt, sondern auch durch die \\ Glaubensrichtung bestimmt.
}

Der individuelle Glaube, die Zugehörigkeit zu einer Glaubensgemeinschaft und die aktive Mitwirkung in dieser Glaubensgemeinschaft stehen für viele Jugendliche nach wie vor in keinem direkten Zusammenhang, sondern werden unabhängig voneinander betrachtet. Vor allem für Adaptiv-Pragmatische, Expeditive und Experimentalistisch-Hedonistische Jugendliche ist es kein Widerspruch, an etwas zu glauben, ohne sich als religiös zu bezeichnen oder als Teil einer Glaubensgemeinschaft zu sehen. Noch am ehesten findet sich der Dreiklang religiöser Praxis bei Konservativ-Bürgerlichen und Sozialökologischen Jugendlichen als zusammenhängendes Konzept: Wer glaubt, gehört einer Religionsgemeinschaft an, und wer einer Religionsgemeinschaft angehört, praktiziert zumindest die zentralen Rituale dieser Religion.

Neben der Zugehörigkeit zu einer Lebenswelt bestimmt aber auch die jeweilige Glaubensrichtung selbst, wie Glaube verstanden und gelebt wird. In den folgenden Abschnitten werden daher insbesondere auch Unterschiede zwischen christlichen und muslimischen Jugendlichen in punkto Religionsverständnis dargestellt. Hier zeigt sich ein unterschiedliches Ausmaß an Selbstverständlichkeit und positiver Identifikation mit dem Glauben sowie eine jeweils anders gelagerte Einbettung religiöser Rituale in den Familienalltag. An dieser Stelle ist aber ein Hinweis auf die Stichprobe bedeutsam, um die Ergebnisse entsprechend einzuordnen: Unter den insgesamt 72 Befragten sind 14 muslimische Jugendliche. Davon besuchen sechs eine Hauptschule und jeweils vier eine Realschule bzw. das Gymnasium. Der Überhang der Hauptschüler unter den muslimischen Jugendlichen ist bei der Interpretation der folgenden Aussagen zu dieser Gruppe zu berücksichtigen. In anderen Worten: Die Befunde zu muslimischen Jugendlichen sagen v. a. etwas über die Muslime aus bildungsbenachteiligten Lebenswelten aus. 


\section{Christliche Jugendliche unterscheiden deutlicher zwischen persönlichem Glauben und Religionszugehörigkeit als muslimische.}

Christliche Jugendliche beschreiben ihren Glauben häufig als eine Art Vertrauen in eine höherstehende Macht, oder als persönliche Strategie, sich mit dem Schicksal zu arrangieren bzw. ihm Sinn zu verleihen. Glaube ist für sie etwas Individuelles, das nicht unbedingt mit einer bestimmten Institution oder Religion verknüpft sein muss. Sie koppeln ihren Glauben in sehr unterschiedlicher Form an konkrete Rituale oder Aktivitäten. Teilweise berichten sie von regelmäßigen Kirchenbesuchen oder der Teilnahme an der kirchlichen Jugendarbeit. Bei anderen Jugendlichen, die ebenso mit dem Glauben verbunden sind, beschränkt sich die Mitwirkung auf den Gottesdienstbesuchen zu Weihnachten und Ostern, oder es werden gar keine Aktivitäten innerhalb der Glaubensgemeinschaft beschrieben.

(1) I glaube ein bisschen an das Schicksal und glaube, dass bestimmte Sachen einfach vorbestimmt sind, weil ich einfach echt schon so krasse Sachen erlebt habe, wo ich dachte, es kann nicht einfach so passiert sein, das muss irgendeinen Hintergrund haben. Aber ich glaube jetzt nicht an Gott oder irgendwie sowas. (weiblich, 17 Jahre, Experimentalistische Hedonisten, christlich)

( Ich glaube an etwas Höherstehendes, ob das jetzt Gott ist oder jemand anderes, lass ich auch offen. Kein Mensch weiß das. Ich glaube an den Himmel, aber ich glaube jetzt nicht an die einzelnen Geschichten. Ich denke schon, dass es einen Menschen gegeben hat, ob der jetzt Jesus hieß oder nicht, und dass der Glauben verbreitet hat und erzählt hat, dass er das Kind Jesu ist. Aber das Ganze mit Moses, der hat das Wasser geteilt und so, das glaube ich jetzt nicht so. Aber ich bin kein Mensch der jetzt so stark einen großen Glauben hat. (männlich, 15 Jahre, Materialistische Hedonisten, keine Religionsgemeinschaft) 


\section{Für das Selbstverständnis muslimischer Jugendlicher spielt Religion oft eine größere Rolle als bei christlichen Jugendlichen.}

Deutliche Unterschiede in der Bedeutung der Religionszugehörigkeit im Alltag gibt es zwischen christlichen und muslimischen Jugendlichen:

Für christliche Jugendliche spielt die Konfessionszugehörigkeit keine besondere Rolle, sie bezeichnen sich häufig ganz allgemein als Christinnen oder Christen. Dies gilt auch, wenn sie an der Firmung bzw. der Konfirmation teilgenommen und sich damit (eigentlich) verbindlich für eine Konfessionszugehörigkeit entschieden haben. Entsprechend werden auch beim Gottesdienstbesuch oder in der Jugendarbeit die Angebote der eigenen Konfession genauso wie die anderer Konfessionen genutzt. Teilweise nimmt man am Religionsunterricht teil, teilweise ist man in das Fach Ethik gewechselt, weil dieses interessanter als der Religionsunterricht erscheint. Christliche Jugendliche sprechen positiv über ihre Religionszugehörigkeit, sind aber bemüht, diese auch immer wieder zu relativieren ("also richtig religiös bin ich nicht"). Sie loten aus, wie weit Religiosität sozial akzeptiert ist; als „streng gläubig“ möchte man keinesfalls wahrgenommen werden.

Typische Zitate zur Illustration

( Ich habe Konfirmation gemacht, aber ich würde mich selbst nicht als religiös bezeichnen. (...) Bis zur 9. Klasse war ich auch evangelisch in der Schule und danach habe ich in Ethik gewechselt. Hat mich mehr interessiert. Ich fand es nicht spannend, irgendwelche Bibelgeschichten zu lesen. In Ethik hat man mehr so philosophische Sachen gemacht, so mit Strafe, Gerechtigkeit und so. Das fand ich viel, viel interessanter. (weiblich, 17 Jahre, Expeditive, christlich)

( Es ist jetzt nicht so, dass wir oder ich jeden Abend beten. Das war früher so. Da haben wir das öfter gemacht. In letzter Zeit fällt mir auf, wir beten nicht mehr so oft vor dem Essen, aber eigentlich machen wir das schon, also so Tischgebet. (weiblich, 16 Jahre, Sozialökologische, christlich)

[INT: WiE IST DAS BEI DIR MIT Religion oder Glauben, IST das EIN WICHTIGes Thema?] Schon. Ich gehe auch in die Kirche. Nicht jede 
Woche, aber hin und wieder auf jeden Fall und natürlich auch an Weihnachten und Ostern und sowas. (männlich, 16 Jahre, Materialistische Hedonisten, katholisch)

I Ich bin evangelisch getauft, und konfirmiert bin ich auch im evangelischen Religionsunterricht. Ich bin aber eigentlich in der katholischen Jugendrunde, weil da viele meiner Freunde sind. Aber wirklich gläubig bin ich nicht. (weiblich, 17 Jahre, Adaptiv-Pragmatische, evangelisch)

Es ist schon wichtig, aber nicht so, dass ich da jetzt alles darauf aufbaue. Aber es ist mir schon wichtig. (weiblich, 17 Jahre, Sozialökologische, katholisch)

Muslimische Jugendliche nennen als Beispiele für die aktive Mitwirkung in ihrer Glaubensgemeinschaft u.a. den Koran-Unterricht, regelmäßige Gebete, den Ramadan, das Zucker- und Opferfest. Auch wenn es hier wie bei den christlichen Jugendlichen eine große Bandbreite der gelebten Zugehörigkeit zur Religionsgemeinschaft gibt, berichten deutlich mehr muslimische Jugendliche über ihre religiöse Praxis im Alltag als Jugendliche anderer Glaubensrichtungen. Viele zeigen eine selbstbewusste, positive und zweifelsfreie Identifikation mit ihrem Glauben, der auch sehr stark im sozialen Zusammenhang der Familie gelebt wird.

[INT: BIST DU RELIGIÖs?] Ja. [INT: WoRAN GLAUBST DU?] An Allah. Ich war auch früher in einem Koran-Kurs. (weiblich, 14 Jahre, Materialistische Hedonisten, muslimisch)

( Ja. Also, ich bin kein strenger Moslem und so, aber ich versuche, ein guter Moslem zu sein. (männlich, 15 Jahre, Konservativ-Bürgerliche, muslimisch)

Interviewer: männlich, 16 Jahre, Materialistische Hedonisten, muslimisch

Also die Frage IST SeHr EINFACH, DENKST DU, DASS Alles UM UNS HeRUM EINEN SCHÖPFER HAT?

( Natürlich. Ohne einen Schöpfer würde die ganze Welt gar nicht entstehen. Die Erde hat auf jeden Fall einen Schöpfer, weil die Erde kann ja nicht einfach so auf die Welt kommen. Alles hat einen Anfang und alles hat ein Ende, würde ich sagen.

Auszüge aus Gesprächen zwischen Jugendlichen zum Thema Glaube und Religion 
IN WELCHER RELIGION BIST DU?

( Ich bin muslimisch und denke, dass es einen Schöpfer gibt namens Allah, und dass die ersten Menschen Adam und Eva sind, und eines Tages wird die Erde auch untergehen. Das heißt in anderen Worten, auch im deutschen Wort, Apokalypse nennt man das.

UND DENKST DU, DASS DIE APOKALYPSE AUCH STATTFINDEN WIRD?

( Natürlich wird das stattfinden. Im Koran wird auch die Apokalypse beschrieben. Sie wird an einem Freitag stattfinden.

WARUM AUSGERECHNET AN EINEM FREITAG? KANN DAS NICHT AUCH AN EINEM SONNTAG ODER AM DONNERSTAG SEIN?

( Die Frage kann ich leider nicht beantworten, aber ich würde eher mal sagen, da die Muslime an einem Freitag in die Moschee gehen. Ja, ich würde sagen, an einem Freitag. Das steht im Koran so geschrieben.

UND DENKST DU, DASS GOTT JETZT ALLES ERSCHAFFEN HAT AUF DIESER WELT?

( Ja natürlich, alles. Alles, von A bis $Z$.

\section{Das Interesse an Sinnfragen ist unabhängig von der Zugehörigkeit zu einer Glaubensgemeinschaft.}

Egal ob muslimisch, christlich oder ohne konfessionelle Zugehörigkeit: Jugendliche interessieren sich für grundlegende Fragen des Lebens allerdings für jeweils unterschiedliche Themen. Während christliche und nicht-religiöse Jugendliche vor allem die Frage bewegt, woher wir kommen und was nach dem Tod kommt, ist für muslimische Jugendliche häufig relevant, was gerecht oder moralisch richtig ist. Hierfür suchen sie teilweise auch Antworten in ihrer Religion.

Typische

Zitate zur Illustration sagen eine Sünde oder so. Da habe ich halt den Imam gefragt, wenn 
man jetzt im Supermarkt arbeitet, ob das jetzt auch so ist. Weil man nimmt ja sozusagen Alkohol, also in dem Geschäft, und verkauft das. Das ist mir einfach so im Kopf steckengeblieben. Da dachte ich, okay, vielleicht ... Da habe ich einfach so gefragt und der meinte dann halt, das wäre für einen Moslem eine Sünde. (männlich, 16 Jahre, Materialistische Hedonisten, muslimisch)

( Es beschäftigt einen schon sehr, auch wenn man selber nicht so gläubig ist. Man denkt halt darüber nach, was ist beispielsweise nach dem Tod, gibt es wirklich einen Gott, der dich dann danach in den Himmel schickt oder nicht. Das sind halt Fragen über Fragen, die alle unbeantwortet bleiben. (männlich, 16 Jahre, Adaptiv-Pragmatische, keine Religionsgemeinschaft)

\section{Für Jugendliche ohne Mitgliedschaft in einer Glaubensgemeinschaft sind Religionen oft interessant und exotisch-reizvoll.}

Jugendliche, die nicht Mitglied einer Glaubensgemeinschaft sind, beschäftigen sich mit den großen Weltreligionen häufig besonders intensiv, da die Zugehörigkeit zu einer Religion für sie nicht selbstverständlich ist. Manche beten auch zu Gott oder einer anderen höheren Macht. GroBes Interesse zeigen diese Jugendlichen für Religionen, die in Deutschland weniger stark vertreten sind, z. B. Buddhismus, Hinduismus oder Judentum. Da Religion für sie nicht qua Familientradition vorgegeben ist, beziehen sie verschiedene Glaubensrichtungen in ihre Auswahl "sympathischer Religionen" mit ein. Hierbei interessieren sie sich für die jeweiligen Dogmen und Überzeugungen, aber mehr noch für den zugehörigen Lebensstil. Jugendliche aus der Prekären Lebenswelt würden Religionen gern einmal kurzfristig und unverbindlich „ausprobieren“, um zu testen, was dahinter steckt, ähnlich wie bei der Auswahl einer neuen Sportart. Experimentalistischen Hedonisten geht es eher um ein aufregendes Experiment, verbunden mit der Suche nach Antworten auf existentielle Fragen und die Erweiterung des eigenen Horizonts. 
Typische Zitate zur |llustration
( Ich bin zwar keine Religiöse, aber ich bete manchmal zu Gott. Ob ich daran glaube oder nicht. (weiblich, 17 Jahre, Prekäre, keine Religionsgemeinschaft)

[INT: GIBT ES EINE GLAUBENSGEMEINSCHAFT, DIE DU INTERESSANT ODER SYMPATHISCH FINDEST?] Interessant sind die eigentlich alle. Die ich am interessantesten finde, das sind die Buddhisten. [INT: WAS FINDEST DU DARAN INTERESSANT?] Die Art und Weise, wie die leben und wie die denken, wie es nach dem Tod ist zum Beispiel, das finde ich interessant. Die ganze Kultur. (weiblich, 16 Jahre, Experimentalistische Hedonisten, keine Religionsgemeinschaft)

(8) Ich mache aber Religionsunterricht in der Schule. Das war anfangs so, weil mich meine Eltern dort angemeldet hatten. Ich mache es auch noch immer, denn ich finde es interessant. Und es ist wichtig, darüber Bescheid zu wissen. Der Religionsunterricht war für mich nie ein Problem, obwohl ich ziemlich sicher bin, dass ich nicht gläubig bin. (weiblich, 15 Jahre, Expeditive, keine Religionsgemeinschaft)

Wir haben auch da unten den Koran stehen. Den habe ich durchgelesen, um herauszufinden, was da so für Zeugs drin steht. (männlich, 17 Jahre, Konservativ-Bürgerliche, keine Religionsgemeinschaft)

Mich interessiert es einfach, wie die leben. Wenn man es selbst erleben kann oder die besuchen kann, das finde ich schon interessant. Das fand ich auch in Thailand so interessant. (weiblich, 16 Jahre, Experimentalistische Hedonisten, keine Angabe zur Religionsgemeinschaft)

( Jetzt bei den Juden würde ich das gerne mal machen. Das würde ich mal gerne machen. [INT: WIESO DAS?] Das interessiert mich, das finde ich toll ... Ich verstehe das auch nicht mit dem Zweiten Weltkrieg und... Und ich mag halt Juden, und wenn ich das so sehe und wie das so immer abläuft, das würde ich auch gerne mal machen. (weiblich, 17 Jahre, Prekäre, keine Religionsgemeinschaft)

(7) Also natürlich, ich finde die Religion alles in allem schon interessant, zum Beispiel den Islam oder so. Natürlich, ich erfahre immer gerne neue Sachen, aber ich glaube, ich könnte das selber nicht, mich an so Sachen halten, Verpflichtungen oder sowas. Das ist schon 
interessant, mehr über die Religion zu erfahren und wie die Leute damit leben. (weiblich, 15 Jahre, Experimentalistische Hedonisten, keine Religionsgemeinschaft)

[INT: WIE IST ES FÜR DICH, MIT DEM MUSLIMISCHEN GLAUBEN IN KONTAKT ZU KOMMEN, WAS DENKST DU DARÜBER?] Ich habe mit anderen Religionen gar keine Probleme, ich finde es interessant. Ich lasse mir da auch gerne was dazu erzählen. Ich komme gerne damit in Kontakt. (weiblich, 16 Jahre, Experimentalistische Hedonisten, keine Religionsgemeinschaft)

\section{Jugendliche ohne Religionszugehörigkeit können sich kaum vorstellen, Mitglied einer Religionsgemeinschaft zu werden.}

Jugendliche ohne Religionszugehörigkeit gehen oft davon aus, dass Religionen sinnstiftend sind und eine Orientierung geben können. Gleichzeitig empfinden sie es als exotisch, an „überirdische Dinge" zu glauben, die unter Umständen gar nicht so stattgefunden haben. „Etwas zu glauben“ bedeutet für sie tatsächlich und konkret an das zu glauben, was in den entsprechenden Schriften steht - und mit Blick auf die gängigen Religionen tauchen dabei für sie große Fragezeichen auf.

Insgesamt überwiegen bei ihnen die Zweifel an der Existenz Gottes und dem Wahrheitsgehalt der Bibel bzw. des Korans, so dass man auch zukünftig nicht Mitglied in einer Glaubensgemeinschaft werden möchte. Gegen die Mitgliedschaft in einer Glaubensgemeinschaft sprechen auch die Regeln, an die man sich dann halten müsste, z. B. Gottesdienstbesuch am Sonntag oder regelmäßiges Gebet. In keinem Fall möchte man von einer Glaubensgemeinschaft missioniert werden. Dies wirkt eher abstoßend.

[INT: Glaubst dU EIgentLICH AN IRGENDETWAS?] Nein, eigentlich nicht wirklich so. Ich kann es mir halt irgendwie nicht vorstellen, dass es da etwas gibt. Das ist irgendwie so unlogisch einfach. (weiblich, Typische Zitate zur Illustration 15 Jahre, Materialistische Hedonisten, keine Religionsgemeinschaft) 
[INT: Und hat das für Dich SCHON MAL EINE Rolle Gespielt, MitGLIED ZU WERDEN IN SO EINER GLAUBENSGEMEINSCHAFT?] Nein. Ich habe mal darüber nachgedacht: Könnte Gott wirklich existieren oder nicht? Na gut, vielleicht schon, aber dann wieder so nee. Also religiös bin ich nicht. (männlich, 14 Jahre, Materialistische Hedonisten, keine Angabe zur Religionsgemeinschaft)

[INT: HAST DU SCHON MAL DARÜBER NACHGEDACHT, MITGLIED IN EINER GLAUBENSGEMEINSCHAFT ZU WERDEN?] Nee, also ich bin irgendwie ... Ich würde nicht sagen, dass ich Atheist bin, aber ich finde das Ganze irgendwie ein bisschen lächerlich. Die Bibel ist für mich eher so ein Märchen als irgendwie Wirklichkeit. (männlich, 17 Jahre, Adaptiv-Pragmatische, keine Religionsgemeinschaft)

Ob sich Jugendliche ohne Zugehörigkeit zu einer Glaubensgemeinschaft für religiöse Themen interessieren, wird wesentlich durch entsprechende Kontaktpunkte im sozialen Nahumfeld bestimmt. Wenn es in der Familie oder im Freundeskreis Menschen gibt, die über ihre Religion und ihren Glauben sprechen, kann dies zu einem interessanten Thema werden. Ebenso bietet der Religions- oder Ethikunterricht Anknüpfungspunkte für die individuelle Beschäftigung mit Glaube und Religion. Fehlen persönliche Kontakte zu Menschen, die über Religion und Glauben sprechen, spielen diese Themen im Alltag kaum eine Rolle.

So ich selber bin in keiner Familie mit Glauben aufgewachsen. Es ist für mich jetzt nicht nachvollziehbar, warum es jetzt wirklich einen Gott geben sollte. Es ist für einen selber dann nicht nachvollziehbar, wenn man da nicht mit reingeboren ist, wenn man darauf nicht erzogen wurde und alles drum und dran. Ja, ich finde an sich Religion teils auch interessant, so wie die dann leben, mit so einer Gemeinschaft in der Kirche. Es ist interessant an sich, aber ich könnte mir da jetzt nichts Ernsthaftes darauf vorstellen, dass es da wirklich einen großen Herrn geben soll, der das dann halt alles gemacht haben soll. Das kann man sich selber nicht vorstellen. Deswegen bin ich da nicht überzeugt. (männlich, 16 Jahre, Adaptiv-Pragmatische, keine Religionsgemeinschaft) 
Interviewer: männlich, 17 Jahre, Konservativ-Bürgerliche, christlich

Wieso GLAUBST DU NICHT AN GOTT?

8 Ich hab mich schon intensiv mit dem Thema auseinandergesetzt, ich habe auch in die Bibel ziemlich intensiv reingelesen. Mir sind diverse Argumente bekannt und zu den meisten davon auch die Konterargumente. Das meiste davon macht keinen Sinn. Es gibt viel zu viele Götter, dass man gar nicht sagen kann, dass einer von denen existieren müsste, weil warum sonst würde man auf so viele kommen. Ich bin zu dem Schluss gekommen, dass Religion einfach nur ein total menschliches Konstrukt ist, das sich in jeder Kultur dann etabliert hat, aber an sich eigentlich nicht stimmt.

UND IST ES SCHON IMMER SO, DASS DU NICHT AN GOTT GLAUBST?

( Ja, und meine Eltern waren auch beide nicht gläubig, haben mich dennoch in den Religionsunterricht geschickt, einfach bloß als Allgemeinbildung. Aber trotzdem bin ich auch, glaube ich, dadurch, dass meine Eltern beide Atheisten sind, dann selber auch zum Atheisten geworden.

Gehst du IN Die KiRChe, VIeLLeicht ZU SPEZIELLEN AnLÄSSEN?

( Ich werde recht oft dazu gezwungen, an Weihnachten in die Kirche zu gehen, weil meine Oma so gläubig ist. Und weil es ihr sehr wichtig ist, tu das. Aber nur sehr, sehr ungern, und ich probiere es zu vermeiden. Und ich glaube, in Zukunft werde ich mich auch da rausreden können, weil ich auch immer älter werde und dann ein bisschen mehr Selbstbestimmungsrecht erhalte.

\section{Die Mitgliedschaft in einer Glaubensgemeinschaft begründet sich in der Regel aus der familiären Tradition.}

Mehrheitlich wurden die Jugendlichen in ihre Glaubensgemeinschaft hineingeboren, im Falle der christlichen Jugendlichen entsprechend der Tradition in Deutschland schon als Baby getauft. Man ist dann ganz selbstverständlich - teilweise auch aus Rücksicht auf die Familie - Mitglied 
der Glaubensgemeinschaft geblieben. Die christlichen Jugendlichen berichten beispielsweise nicht davon, dass Firmung oder Konfirmation ein Anlass gewesen wäre, den eigenen Glauben zu überprüfen. Vielmehr bestärken andere einschneidende Ereignisse, die man (mit)erlebt oder antizipiert (wie z. B. Hochzeit oder Tod), die Zugehörigkeit zur Glaubensgemeinschaft.

Typische Zitate zur |llustration

Ich bin katholisch. Katholik. [INT: WIE KAM ES DAZU?] Keine Ahnung. Meine Eltern haben das gesagt. [INT: DU WURDEST ALS BABY GETAUFT?] Ja. Ich wurde getauft und dann wurde mir gesagt, Konfirmation musst du machen, weil das sieht ja komisch aus, wenn die Enkelin von den Kirchenchorvorständen nicht konfirmiert wird. Okay, dann mache ich das halt. (weiblich, 17 Jahre, Konservativ-Bürgerliche, evangelisch)

I Ich komme ja aus Russland. Da ist halt einfach jeder Christ, und da wirst du halt auch getauft, kriegst du dein Kreuz, gehst dann so ab und zu mal in die Kirche mit deiner Oma so. Ja und bist dann halt einfach gläubig so. (männlich, 15 Jahre, Prekäre, russisch-orthodox)

[INT: UND WIE KAM ES DAZU? ODER WAR DAS SCHON IMMER SO, DASS DU AN AlLah GEGLaUbT HAST?] Meine Eltern kommen aus der Türkei und die glauben ja. Also wir glauben alle an ihn. [INT: UND DAS KAM DANN QUASI SO AUTOMATISCH?] Ja. [INT: UND IST DER GLAUBE EURER GANZEN FAMILIE WICHTIG?] Ja. (weiblich, 14 Jahre, Materialistische Hedonisten, muslimisch)

( Wir sind Aleviten und halt zwischen Aleviten und Sunniten gibt es sehr viel Streit in der Türkei. [INT: ABER WOHER KAM DAS ZUM BEISPIEL MIT DEM Glauben?] Durch meine Familie alles. Also wir sind jetzt nicht extrem gläubig, aber schon ... (weiblich, 16 Jahre, Adaptiv-Pragmatische, muslimisch)

Jugendliche, in deren familiärem Umfeld Religion und Glaube kaum eine Rolle spielen, sind überwiegend konfessionslos. Von einer eigenen „bewussten“ Entscheidung, Mitglied einer Glaubensgemeinschaft zu werden, berichten nur wenige Jugendliche. Sie ist dann in der Regel durch aktives kirchliches Engagement (z. B. in der konfessionellen Jugendarbeit) oder einen konkreten Anlass (z. B. Heirat) bedingt. 
[INT: DANN HAST DU JA GESAGT, DASS DU KONFIRMIERT BIST. WIE BIST DU DENn Mitglied einer Glaubensgemeinschaft GeWORDEN? EINFACH WEIL DU GETAUFT WURDEST, ODER HAST DU DICH SELBST DAFÜR ENTSCHIEDEN?] Ich habe mich selbst dafür entschieden. Das kam aber mehr oder weniger dadurch, dass ich evangelisch wurde, weil ich eben auch schon früher auf so evangelischen Jugendfreizeiten dabei war. (männlich, 16 Jahre, Adaptiv-Pragmatische, evangelisch)

(7) [int: Kommt Dein Glaube durch Deinen Freund?] Ja. Seine Mutter hat mir mal gesagt, dass, wenn ich das will, ich mal mitgehen kann. Und seitdem finde ich das richtig gut. (weiblich, 16 Jahre, Materialistische Hedonisten, evangelisch)

( In der Familie meiner Oma gab es einige Pfarrer. Ich weiß aber gar nicht, ob meine Großeltern gläubig sind. Es spielte bei uns nie eine große Rolle. Wir sind auch Weihnachten nie in die Kirche gegangen. (weiblich, 15 Jahre, Expeditive, keine Religionsgemeinschaft)

\section{Auch bei geringer Religionsverbundenheit zeigen die christlichen Jugendlichen keine ausgeprägte Bereitschaft zum Kirchenaustritt.}

Die christlichen Jugendlichen wägen auf die Frage nach einem möglichen Kirchenaustritt verschiedene Argumente für den Ausstieg und den Verbleib in der Glaubensgemeinschaft bzw. der Kirche gegeneinander ab. Wenige fühlen sich mit der eigenen Konfession so stark verbunden, dass ein Kirchenaustritt gar nicht zur Diskussion steht. In der Regel haben die Jugendlichen aktuell einfach keinen Anlass, die Glaubensgemeinschaft zu verlassen bzw. aus der Kirche auszutreten. Einzelne Jugendliche haben sich jedoch bereits dazu entschieden, später aus der Kirche auszutreten, allerdings aus unterschiedlichen Motiven: Von den Expeditiven wird als Argument vor allem mangelnde Beteiligung am Kirchenleben oder das fehlende Interesse am bzw. die Distanz zum Glauben genannt. Der finanzielle Aspekt der Kirchensteuer wird am ehesten von Adaptiv-Pragmatischen und Hedonistischen Jugendlichen angeführt. Da man aktuell aber noch keine Kirchensteuer zahlt, kann man mit dem Austritt aber noch 
warten. Man ist außerdem oft zu bequem, sich über das bürokratische Verfahren zu informieren. Konservativ-Bürgerliche und Sozialökologische Jugendliche denken am wenigsten über einen Kirchenaustritt nach.

\section{Typische}

Zitate zur Illustration

[INT: WÜRDEST DU AUCH AUS DER KIRCHE AUSTRETEN?] ICh kenne mich nicht genau mit diesen Kirchensteuern aus. Die würde ich nicht unbedingt zahlen wollen, weil ich eben nicht wirklich gläubig bin. Aber das hat ja noch Zeit, bis ich 18 bin. (weiblich, 17 Jahre, Adaptiv-Pragmatische, evangelisch)

(7) [INT: Aber Willst du denn aus der KiRCHe austreten?] Ja, auf jeden Fall. Also könnte ich jetzt, glaube ich, sogar schon machen, aber ich habe mich jetzt auch nicht so genau informiert. Aber auf jeden Fall werde ich das machen. (weiblich, 17 Jahre, Expeditive, christlich)

( Die Vorstellung der Kirche finde ich super. Ich finde es toll, dass man dort seinen Glauben leben kann. Aber das kann man auch an anderen Plätzen, an komplett anderen Stellen. Zum Beispiel zu Hause für sich oder mit anderen Leuten. Es geht auch anders. Man muss da keine Steuern für zahlen. Es ist klar, dass man so etwas finanzieren muss. Aber halt auch nicht so hoch. Vielleicht sollte man die Steuer ein wenig senken, dann hätte die Kirche auch wieder mehr Zulauf. Auf jeden Fall habe ich darüber nachgedacht, weil ich nicht daran glaube. Ich gehe immer noch gern in Kirchen, und ich bete auch noch. Und ich zünde auch noch Kerzen an. Und ich werfe immer eine Spende hinein. Noch muss ich nicht mein Geld bezahlen. Meine Mutter hat gesagt, ich kann austreten, wenn ich das möchte. Aber ich wollte mich eigentlich noch firmen lassen, weil sich das meine Oma gewünscht hat. Aber ich möchte nichts vorgaukeln, nur um es meiner Oma recht zu machen. Ich stecke da irgendwie in einem Zwiespalt. Ich weiß es nicht. Ich glaube an Gott, aber irgendwie auch nicht. Ich kann aber nicht mit gutem Gewissen sagen, dass ich total fest an Gott glaube. Das könnte ich nicht. Denn ich weiß genau, das stimmt nicht. Ich möchte niemandem ins Gesicht lügen. (weiblich, 16 Jahre, Experimentalistische Hedonisten, christlich)

Das Engagement in der Jugendarbeit und der Freundeskreis haben einen positiven Einfluss auf den Verbleib in der Glaubensgemeinschaft. Vor 
allem in traditionellen und bürgerlichen Lebenswelten tragen konkrete Gemeinschaftserfahrungen in der Jugendkirche dazu bei, der Glaubensgemeinschaft treu zu bleiben. Konservativ-Bürgerliche Jugendliche formulieren eindeutig, dass sie Mitglied der Kirche bleiben, solange sie weiter an den Gottesdiensten teilnehmen.

Für „unentschiedene" Jugendliche spielen für den Verbleib in der Glaubensgemeinschaft u.a. die Intensität der Auseinandersetzung mit dem eigenen Glauben, familiäre Gründe oder auch der Wunsch nach einer kirchlichen Hochzeit eine Rolle. Diese Aspekte haben oft ein größeres Gewicht als mögliche Austrittsgründe.

[INT: HAST DU SCHON MAL DRAN GEDACHT, AUS DER KIRCHE AUSZUTRETEN?] Nein, überhaupt nicht. Ich sehe eigentlich keinen Grund. Ich finde die evangelische Kirche auch besser als die katholische. Die Ansichten von manchen Katholiken, wenn jemand so streng gläubig ist, das finde ich manchmal schon ein bisschen krass auch, aber auch ein bisschen dumm einfach. (weiblich, 16 Jahre, Expeditive, evangelisch)

[INT: HAST DU SCHON MAL DARÜBER NACHGEDACHT, AUSZUTRETEN?] Nee. Ich gehe ja an Weihnachten in die Kirche. (weiblich, 17 Jahre, Konservativ-Bürgerliche, evangelisch)

[INT: HASt Du SCHON MAL DARAN GEDACHT, AUS DER KIRCHE AUSZUTRETEN?] Nein, ich denke, man muss ja nicht aus der Kirche austreten, um nicht mehr den extremen Glauben zu haben. Man kann ja trotzdem in der Kirchengemeinde bleiben. Aber man braucht ja nicht der Engagierteste sein. (weiblich, 14 Jahre, Adaptiv-Pragmatische, katholisch)

[INT: HAST DU SCHON MAL DRÜBER NACHGEDACHT, AUSZUTRETEN?] Ich habe schon mal drüber nachgedacht. Ich gehe nie in die Kirche. Ich habe mit so Christentum und so eigentlich nicht viel am Hut. Das Einzige, was ist, wenn man später mal heiratet oder so, dann will man vielleicht doch kirchlich heiraten. Aber das wäre ja auch doof, deshalb alleine in der Kirche zu bleiben, wenn man keine Überzeugung dazu hat. Das muss ich mir noch überlegen. (weiblich, 17 Jahre, Expeditive, evangelisch) 
Selbst wenn sie sich wenig mit Fragen der Religion und des Glaubens beschäftigen, erkennen viele Jugendliche an, dass Glaubensgemeinschaften wichtig sein können, insofern sie Menschen verbinden und Sinn stiften.

\subsection{Glaube und Religion im Alltag}

\section{Religiöse Praxis ist oft nicht im Alltag verankert und auf bestimmte Anlässe beschränkt.}

Die religiöse Praxis der meisten Jugendlichen ist überwiegend anlassbezogen und findet zu spezifischen Zeiten im Jahresverlauf (Weihnachten, Ramadan, Ostern, Zuckerfest etc.) und in bestimmten Lebensabschnitten (z. B. Konfirmandenzeit) statt. Zudem gibt es einzelne Momente, in denen die Zugehörigkeit zu einer Religionsgemeinschaft relevant wird; dazu gehören beispielsweise Krisensituationen, in denen man Unterstützung und Entlastung "von irgendwoher" sucht, aber auch zentrale Lebensfragen („Was kommt nach dem Tod?") oder der Gedanke an die spätere Heirat.

Am häufigsten wird das Gebet als religiöse Praxis erwähnt. Für manche gehört das regelmäßige Gebet zum Alltag, noch häufiger wird jedoch in Krisensituationen und vor besonderen Herausforderungen gebetet. Das so bezeichnete "Stoßgebet" gibt das Gefühl, dem Schicksal einen Schubs geben zu können und verschafft eine gewisse Entlastung. Bei positivem Ausgang der Krise bedankt man sich dann auch entsprechend (z.B. bei Gott). Insbesondere im Alltag der Materialistisch-Hedonistischen Jugendlichen christlichen und muslimischen Glaubens ist das Gebet eine häufig genannte religiöse Praxis, die durchaus mit der Hoffnung auf konkrete Hilfe durch Gott verbunden ist.

Der regelmäßige Besuch der Kirche oder der Moschee spielt dagegen eine eher untergeordnete Rolle. Viele Jugendliche berichten jedoch, dass sie an den Feiertagen - Weihnachten und Ostern, Zucker- und Opferfest - oder im Kontext ihrer eigenen Erstkommunion, Firmung oder Konfirmation 
die Kirche bzw. Moschee besuchen bzw. besucht haben. Die Teilnahme an den Gottesdiensten ist dabei meist mit Familienfeiern zu den entsprechenden Anlässen verbunden. In der Wahrnehmung der Jugendlichen sind Gottesdienst und ritualisiertes Gebet damit eher Bestandteil des Familienlebens als bewusste Teilnahme am religiösen Leben.

(1) [INT: WAS FüR EINE BEDEUTUNG HAT DAS Für DICH?] Na ... Einfach so das ... Wenn jetzt irgendwie so was passiert, was jetzt nicht so toll ist, denke ich an Gott so ... Wenn irgendwie was Tolles passiert ist, danke ich Gott, oder so. (männlich, 15 Jahre, Prekäre, russisch-orthodox)

Typische

[INT: UND WAS WÜRDEST DU SAGEN, WIE ZEIGT SICH DEIN GLAUBE IM AlLTAG?] Ich weiß nicht, vielleicht auch einfach so in der Lebenseinstellung, dass man so ein bisschen danach lebt, wird schon richtig sein. Oder in einer brenzligen Situation oder wenn man aufgeregt ist vor so einer Debatte, dass man einfach kurz so ein Stoßgebet zum Himmel schickt oder dass man sagt, ja, Gott wird schon meine Worte irgendwie kommen lassen, und wenn es nicht klappt, dann war es auch für etwas gut. Einfach so, dass man alles ein bisschen lockerer sieht vielleicht. [INT: UND DAS HILFT DANN AUCH?] Ja, schon. (weiblich, 16 Jahre, Sozialökologische, katholisch)

[iNT: Und dein Glauben, Wie zeigt der sich im Alltag?] Dass man zum Beispiel, wenn man betet oder sowas. Also mir persönlich, ich fühle mich danach befreiter. Und im Alltag, da würde ich sagen, man denkt schon mehr über manche Sachen nach, finde ich. (männlich, 16 Jahre, Materialistische Hedonisten, katholisch)

( Klar, wenn man irgendwie ein bisschen traurig ist oder so, dann kommt es schon mal vor, dass man irgendwie mal in sich geht und mal wirklich irgendwie versucht, irgendwie zu beten oder so, aber sonst halt irgendwie beten oder so tue ich auch wirklich nicht allzu oft. Ja, sonst halt eigentlich nur, wenn man irgendwie, also Ostern, Weihnachten, das sind so die Feste, wann man noch so in die Kirche geht, ehrlich gesagt. Aber ich glaube, ich werde später auch öfter noch mal in die Kirche gehen, wenn ich halt dann irgendwie etwas älter bin. (männlich, 17 Jahre, Experimentalistische Hedonisten, katholisch) 
Kircheliche Jugendarbeit, die Tätigkeit als Ministrant oder Ministrantin oder der Besuch einer Jugendkirche werden von christlichen Jugendlichen als weitere Möglichkeiten der Mitwirkung im Alltag der Glaubensgemeinschaft genannt. Dabei wird jedoch selten ein unmittelbarer Bezug zum eigenen Glauben oder zum eigenen Alltag hergestellt.

\section{Bei muslimischen Jugendlichen hat die Religion eine stärkere Orientierungsfunktion im Alltag.}

Insgesamt fällt bei den muslimischen Jugendlichen im Vergleich zu den Äußerungen der christlichen Jugendlichen auf, dass Regeln in Form religiöser Ge- und Verbote stärkeres Gewicht für den Alltag haben, so dass Religion teilweise auch als moralisches Grundgerüst für den Alltag dient, während die christlichen Jugendlichen bei ihrer Religion eher nach Bedarf Orientierung und Unterstützung "abrufen“ - sei es in punkto Entscheidungshilfe oder für die erfolgreiche Bewältigung von Aufgaben.

Vor allem muslimischen Jugendlichen gibt Religion darüber hinaus auch Orientierung für das Handeln im Alltag; Religion hilft ihnen, die „richtigen" Entscheidungen zu treffen, zum Beispiel durch die Teilnahme am Freitagsgebet oder religiösen Feiern oder den Austausch mit Geistlichen oder anderen Gläubigen. Zumeist beschreiben sie aber ihren Glauben als allgemeine Lebenseinstellung, die sie im Alltag kontinuierlich begleitet.

( Ich bete normalerweise. Man hat fünf Mal am Tag Gebete. Dann hat man den Ramadan, an dem man fastet. Dann hat man bestimmte Tage im Jahr, wo dann besondere Tage sind. Dann hat man die Feste, zwei große Feste: Zuckerfest und Opferfest. Dann eigentlich allgemein, auch wie ich rede, hat auch etwas mit dem Islam zu tun, weil ich sehr viele Wörter auch permanent Gott danke sozusagen. So Kleinigkeiten sind eigentlich immer im Alltag mit drin, auch wenn ich gerade nicht bete. (weiblich, 16 Jahre, Konservativ-Bürgerliche, muslimisch)

Muslimische Jugendliche nennen als weitere Beispiele ihrer religiösen Praxis bzw. als Erkennungsmerkmale ihrer Religion auch den Verzicht auf Alkohol und Schweinefleisch oder das Tragen eines Kopftuches. 
( Ich bin halt in der Kirche aktiv. Also ich bin halt für so Zeltlager, für Veranstaltungen für Kinder bin ich halt dabei und helfe. Aber das mache ich auch nur, weil ich da Freunde habe. Jetzt zum Beispiel fahren wir morgen ins Zeltlager. Da lassen wir die Kinder spielen und alles mögliche. (männlich, 16 Jahre, Adaptiv-Pragmatische, katholisch)

( Als Moslem ist es ja auch wichtig, gläubig weiterzuleben. Und wenn ich jetzt eine Person heiraten würde, die jetzt nicht gläubig ist, dann würde es ja sozusagen so eine Linie zwischen uns sein. Einer ist gläubig, der andere ist nicht gläubig. Dann ist es dann immer so komisch, weil man soll ja zusammen etwas machen. Wenn ich jetzt beten würde und meine Frau zum Beispiel jetzt nicht betet oder sie sitzt da und spielt mit dem Handy, ich bete und dann... Einfach, das ist dann im Zusammensein ein anderes Gefühl, wenn man zusammen betet. Dann fühlt sich das immer schöner an. (männlich, 16 Jahre, Materialistische Hedonisten, muslimisch)

Auf meinen Alltag wirkt sich mein Glaube sehr aus. Durch meinen Glauben kann ich ja auch wissen, was ich mache. Also das, was ich mache, ob das richtig ist oder nicht. Das ist dann auch sehr wichtig. Dass man keine schlechten Sachen und so machen soll, ja. (männlich, 14 Jahre, Prekäre, muslimisch)

[INT: GIBT ES NOCH ETWAS, WORAN MAN ERKENNEN KANN, DASS DU Mitglied deINer GemeInde BIST?] Ja, ich trage ein Kopftuch normalerweise. Jetzt habe ich zwar grade keines an, weil ich zu Hause bin und meine Religion ist nicht... Also meine Religion akzeptiert es, dass ich mich da ohne Kopftuch zeige sozusagen. Aber sonst trage ich ein Kopftuch. (weiblich, 16 Jahre, Konservativ-Bürgerliche, muslimisch) 


\section{Seinen Glauben zu leben hat für Jugendliche in erster Linie etwas mit der Einhaltung von Regeln, aber auch mit positiven Gemeinschaftserlebnissen zu tun.}

Obwohl muslimische Jugendliche ihren Glauben zum Teil stärker im Alltag verankern, können christliche und muslimische Jugendliche gleichermaßen nichts mit dem Begriff "den Glauben leben" anfangen. Die in Glaubensgemeinschaften verbreitete Idee eines im Alltag spürbaren und lebendigen Glaubens können viele Jugendliche nicht auf sich selbst beziehen. Der Beginn des Kapitels zeigte zwar, dass einige christliche und muslimische Jugendliche ihren Glauben in Ansätzen in den Alltag integrieren, allerdings werden bei dieser Art der Umschreibung die entsprechenden Bilder nicht aufgerufen. "Seinen Glauben leben“ wird eher als Appell verstanden und ruft vornehmlich ein formales Verständnis von Glaubensritualen auf.

Man denkt dabei vorrangig an Gebete, Gottesdienst- oder Moschee-Besuche sowie die Einhaltung von vorgegebenen Verhaltensregeln der jeweiligen Religion, z.B. Teilnahme am Ramadan, Einhaltung der Zehn Gebote oder Nächstenliebe. Dies gilt gleichermaßen für Jugendliche, die sich einer Glaubensgemeinschaft zugehörig fühlen wie für die anderen. Man assoziiert damit also eher Verpflichtungen oder Einschränkungen des persönlichen Handelns als individuelle Aneignungsprozesse religiöser Praxis.

Am ehesten würden christliche und muslimische Konservativ-Bürgerliche und Materialistische Hedonisten von sich sagen, dass sie "ihren Glauben leben". Im Alltag von Expeditiven und ExperimentalistischHedonistischen Jugendlichen spielen religiöse Praktiken und Regeln die geringste Rolle. Auch christliche Materialistische Hedonisten und Prekäre behaupten kaum von sich, dass sie ihren Glauben leben. Sie betrachten Religion eher funktional und nutzen sie, wenn entsprechender Bedarf besteht. 
[INT: HAST DU SCHON MAL GEHÖRT, DASS MAN SAGT, SEINEN GLAUBEN LEBEN?] Ja. [INT: UND TRIFFT DAS AUF DICH zU?] Nicht wirklich. Also ich lebe so wie ich gerade Lust habe. (weiblich, 17 Jahre, KonservaTypische Zitate zur |llustration tiv-Bürgerliche, evangelisch)

( Ich nehme mir jetzt nicht wirklich viel Zeit, ich würde jetzt nicht sagen, dass ich hochgläubig bin, aber einfach so ... Glauben leben ... Naja, nicht unbedingt ... Ab und zu mal in die Kirche aber sonst nicht irgendwelche besonderen Rituale oder so, das jetzt nicht. (männlich, 16 Jahre, Adaptiv-Pragmatische, evangelisch)

( I I hätte gar nicht die Zeit dazu, jeden Sonntag zur Kirche zu gehen und unter der Woche verschiedene Aktivitäten in der Kirche zu machen. Dazu hätte ich gar nicht die Zeit. Aber ich habe auf jeden Fall gar nichts dagegen, wenn verschiedene Leute das so machen, wie sie es wollen. Wenn andere muslimisch sind und einen anderen Glauben haben, dann habe ich auch nichts dagegen. (männlich, 16 Jahre, Materialistische Hedonisten, katholisch)

(1) Ja zum Beispiel, wenn in der Religion steht, du darfst keinen Alkohol trinken, du darfst das nicht, das nicht, dann halt das nicht tun. Das ist so etwas. (männlich, 17 Jahre, Materialistische Hedonisten, muslimisch)

[INT: HAST DU SCHON MAL GEHÖRT, DASS MAN SEINEN GLAUbEN LEBEN KANN? HAST DU DAZU EINE VORSTELLUNG?] Ich weiß nicht, wenn man kein Fleisch oder so isst, weil es einem die Religion verbietet oder so etwas, das kann ich mir vorstellen. Und es gibt doch auch Buddhismus, dass die da irgendwie so Lebensweisheiten haben und so, dass die das sozusagen leben, was Buddha gesagt hat. (weiblich, 15 Jahre, Materialistische Hedonisten, keine Religionsgemeinschaft)

[INT: HAST DU SCHON MAL DEN BEGRIFF GEHÖRT "GLAUBEN LEBEN"?] Nicht gehört, aber ich könnte mir da etwas drunter vorstellen. Also, unter den Gesetzen von diesem Gott leben. Zum Beispiel dieses Ramadan, was da letztens auch wieder war. (weiblich, 15 Jahre, Sozialökologische, keine Religionsgemeinschaft)

Die Auseinandersetzung mit dem Thema Glauben im Alltag zeigt deutlich auf, dass vielen Jugendlichen die kirchlichen Begrifflichkeiten fremd 
oder restriktiv erscheinen. In ihren eigenen Worten berichten sie aber ausführlich darüber, wie sich ihr Zugehörigkeitsgefühl zu einer Glaubensgemeinschaft und die Verankerung religiöser Praxis im Alltag durch konkrete Gemeinschaftserfahrungen verstärkt. Diese sozialen Motive für religiöse Praxis stehen für viele Jugendliche eindeutig im Vordergrund, werden aber nicht unmittelbar mit dem persönlichen Glauben oder der Formulierung "seinen Glauben zu leben" in einen Zusammenhang gestellt, sondern für sich genommen als wertvoll beurteilt und als wesentliche Triebfedern religiöser Alltagsrituale gesehen.

( Ja, da sind halt alle, die man kennt. Das ist so ein kleiner Kreis. Und da bringt auch immer jemand Kuchen mit. Und den essen wir dann. Und dann predigt einer. Und dann ist da manchmal eine Taufe oder so etwas. Das ist richtig cool. (weiblich, 15 Jahre, Materialistische Hedonisten, evangelisch)

( Man kann auch zu Hause beten und so, aber wenn man in der Moschee betet, ist es noch etwas anderes, schöner und auch besser. (männlich, 16 Jahre, Konservativ-Bürgerliche, muslimisch)

(2)il im Islam ist es ja so, da hat man seinen Bund, dieses, wie soll ich das erklären, dieses Miteinander. Man ist immer mit anderen Jugendlichen oder mit anderen islamischen Gruppen zusammen. Das finde ich voll gut. Das mag ich voll. (männlich, 16 Jahre, Materialistische Hedonisten, muslimisch)

Vereinzelt wird auch allein die Möglichkeit, seine Religion ausüben zu dürfen, ohne dafür verfolgt zu werden, als "den Glauben leben“ verstanden.

[INT: InWIefern tRIfFT die Aussage "Seinen Glauben leben“ auF $\mathrm{DICH}$ ZU?] Dass ich in meinem Land nicht verfolgt werde, wenn ich sage, dass ich Christ bin. Dafür bin ich sehr dankbar. Und dass ich mich offen mit anderen Christen treffen kann. Dass ich in die Kirche gehen darf. Und dass ich in der Bibel lesen kann. Und dass ich beten kann. (männlich, 15 Jahre, Sozialökologische, christlich) 


\subsection{Leben mit religiöser Vielfalt}

\section{Die Freundeskreise der Jugendlichen sind oft religiös heterogen.}

Für die meisten Befragten ist es Normalität, dass sie in ihrem Freundeskreis Jugendliche verschiedener Religionen, aber auch Konfessionslose haben. Besonders häufig werden verschiedene christliche Konfessionen, der Islam sowie keine Religionszugehörigkeit genannt, aber auch der Buddhismus und das Judentum werden erwähnt. Oftmals kennt man die Religionszugehörigkeiten der Freundinnen und Freunde gar nicht genau, da sie keine große Bedeutung für die Freundschaft haben. In keinem Fall stehen unterschiedliche Religionszugehörigkeiten einer Freundschaft im Weg. Die religiöse Vielfalt im Freundeskreis steht in engem Zusammenhang mit der Vielfalt der Nationen und Kulturen der Freundinnen und Freunde. Das lässt sich, mit Ausnahme der Materialistisch-Hedonistischen und der Prekären Lebenswelt, milieuübergreifend verallgemeinern.

(7) [iNT: Wie sieht es in Deinem Freundeskreis aus? GibT es da VerSCHIEDEne ReLigionen?] Manche sind halt muslimisch. Und manche sind evangelisch. Aber eigentlich ist das alles gemischt, und es wird Typische Zitate zur Illustration auch kein Wert darauf gelegt. Es wird nicht darauf geachtet. (weiblich, 15 Jahre, Adaptiv-Pragmatische, katholisch)

(7) [INT: WIE IST ES IN DEINEM FREUNDESKREIS, SIND DA IRGENDWELCHE Religionen Vertreten?] Also ein paar meiner Freunde sind christlich. Meine Patentante ist Muslimin. Ich kenne auch halt einfach von der Familie, ein paar Bekannte sind Türken. Und ich habe da wirklich nichts gegen. Ich akzeptiere jeden vom Glauben her. Mein Urgroßvater war Jude und durch die Religion wäre ich ja theoretisch auch Jüdin. Ich verstehe das alles und finde das auch nicht schlimm. Ich kenne auch einige Muslime, ich kenne ein paar Christen, ich kenne glaube ich gar keine Juden. Aber ich habe aber niemanden so im Freundeskreis, der komplett streng gläubig ist, der wirklich jeden Sonntag in die Kirche geht. (weiblich, 17 Jahre, Adaptiv-Pragmatische, keine Religionsgemeinschaft) 
( Also man hat ja generell Freunde aus allen Religionen. (männlich, 16 Jahre, Materialistische Hedonisten, katholisch)

(7) [inT: Welche Religionen GibT ES Denn noch so bei DiR im FreunDESKREIS?] Ich habe einen Freund, der ist jüdisch. Und mehrere Freunde sind Muslime. Und ansonsten gibt es noch Atheisten, aber das kann man nicht wirklich als Religion definieren. Also es ist ziemlich bunt gemischt. [INT: UND WIE KOMMT IHR SO MITEINANDER KLAR MIT DEN UNTERSCHIEDLICHEN Religionen?] Das spielt bei uns gar keine Rolle. Das bleibt außen vor. Da steht nichts im Weg. (männlich, 15 Jahre, Sozialökologische, christlich)

( [INT: GibT es Unterschiedliche Religionen beI diR IM FreunDESKREIS?] Ja. Ich weiß nicht, wie man diese ausländischen Religionen nennt. Da sind diese ausländischen Leute. [INT: UND WELChe Religionen die haben?] Das weiß ich jetzt nicht. Katholisch oder keine Ahnung. (männlich, 14 Jahre, Prekäre, keine Angabe zur Religionszugehörigkeit)

Bei den Materialistischen Hedonisten und den Prekären zeigen sich zum Teil gegenläufige Tendenzen: Ein Teil dieser Jugendlichen berichtet von einer großen religiösen Vielfalt im Freundeskreis. Andere Jugendliche dieser Lebenswelten, vor allem solche muslimischen Glaubens, berichten aber, dass ihre Freunde alle den gleichen Glauben haben (siehe auch das Kapitel zu Nation und nationaler Identität). Dabei spielt auch eine engere Einbindung in das familiäre Umfeld eine Rolle.

Dort, wo die religiöse Vielfalt im lokalen Umfeld geringer ist, z. B. in ländlichen Regionen oder in den ostdeutschen Bundesländern, ist auch die religiöse Vielfalt in den Freundeskreisen geringer. Die Unterschiede beschränken sich dann auf gläubig und nicht-gläubig, verschiedene christliche Konfessionen oder vereinzelte Jugendliche anderer Religionen, v. a. muslimische Jugendliche. 


\section{Der Austausch über unterschiedliche Religionen spielt im Alltag eine Nebenrolle.}

Religion und Glauben gehören nicht zu den Themen, über die man im Alltag viel mit seinen Freundinnen oder Freunden spricht oder für die man sich besonders interessiert, auch wenn die Freundinnen oder Freunde einer anderen Glaubensgemeinschaft angehören. Vereinzelt stellt man Fragen an Jugendliche anderer Glaubensrichtungen, oder man bekommt hin und wieder von religiösen Feierlichkeiten und Praktiken erzählt. Auch der schulische Religionsunterricht kann ein Anlass sein, sich über Glauben und Religion auszutauschen.

(7) [INT: Führt IHR manchmal Diskussionen ÜBer Religion UND Glaube?] Nein, eigentlich nicht so. Ich habe auch einige Freunde, die unreligiös sind. Die haben einfach ein paar Fragen gestellt und nicht mehr. (weiblich, 14 Jahre, Adaptiv-Pragmatische, muslimisch)

(7) [INT: WIE SieHt es IN DeInem Freundeskreis aus?] Meine Freunde sehen es eigentlich genauso wie ich. Sie befolgen die Religion auch nicht. Es gibt natürlich diese Leute, die den Muslimen angehören. Die müssen dann auch mal fasten oder so. Das kann ich verstehen. Aber ich selber befolge solche Regeln nicht. Und sehr viele meiner Freunde tun das auch nicht. (männlich, 16 Jahre, Adaptiv-Pragmatische, orthodox)

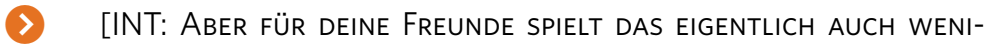
GER EINE ROLLE ODER GIBT ES JEMANDEN, FÜR DEN DAS WICHTIG IST, DER GLÄUBIG IST?] Ja, ich habe halt ein paar Freunde, die sind streng gläubig. Das sind meistens die Muslime. Die müssen das halt folgen. Aber der Rest ist eher so wie ich. Das ist jetzt nicht so wichtig für die. [INT: WIE DRÜCKT SICH DAS BEI DENEN AUS, DASS IHNEN DAS WICHTIG IST? ERZÄHLEN DIE EUCH AUCH DARÜBER WAS?] Nee, also die behalten das lieber für sich. Ich frage auch nicht so oft nach. [INT: ALSO DARÜBER SPRECHT IHR EIGENTLICH NICHT?] Nee. (männlich, 16 Jahre, Adaptiv-Pragmatische, katholisch)

Am ehesten sind Glaube und Religion bei den postmateriell geprägten und den postmodernen Lebenswelten (Sozialökologische, Expeditive, 
Experimentalistische Hedonisten) ein Gesprächsthema im Freundeskreis. Das sind die Lebenswelten, die sich besonders kritisch mit Glaube und Religion auseinandersetzen und zugleich ein besonderes Interesse an der Vielfalt der Kulturen und Religionen haben. Solange die persönlich vertretenen Werte, wie z. B. Toleranz, nicht in Konflikt mit einer Religion bzw. dem jeweiligen Glauben treten, zeigt man sich offen und interessiert, andernfalls kann aber auch die Freundschaft in Frage gestellt werden.

(7) Eine Freundin von mir ist ziemlich gläubig. Sie hat aber trotzdem nichts gegen Homosexuelle oder Adoptionen oder Abtreibungen. Sie ist also sehr tolerant. Anders finde ich es sehr schwierig. Wir haben zum Beispiel ein Mädchen in der Klasse, die sehr gläubig ist. Sie ist auch so ein Mensch, die sagt: "Ich habe zwar nichts gegen Homosexuelle, aber eigentlich ist es doch nicht das Richtige." Und mit solchen Leuten kann ich nicht wirklich reden. Sie argumentiert dann immer mit den Worten: "Das ist mein Glaube, und dagegen kannst Du nichts sagen." Dabei will ich gar nichts gegen den Glauben sagen. Ich will nur etwas dagegen sagen, dass sie offenbar etwas gegen Homosexuelle hat. (weiblich, 15 Jahre, Experimentalistische Hedonisten, keine Religionsgemeinschaft)

Für Jugendliche, die keiner Glaubensgemeinschaft angehören, ist es besonders interessant, dass bei gläubigen Jugendlichen der Alltag durch den Glauben geprägt ist, oder dass diese von rational unerklärlichen Phänomenen berichten, z. B. vom Kontakt mit Verstorbenen oder von Vorzeichen für Unglück an einem bestimmten Tag.

[INT: Wie ZEIGEN DAS DEINE FreUnde denN IM AlLtAG, DASS SIE GLÄUBIGE MENSCHEN SIND?] Mein bester Freund hat einmal gesagt: Mein Koran ist runter gefallen, und dann hatte ich an dem Tag die ganze Zeit Pech gehabt. So etwas zum Beispiel. [INT: WeLCHe Rolle SPIELT DAS IN SEINEM AlLTAG?] Ich denke mal, er ist nicht so extrem gläubig, aber er glaubt schon. Er betet halt auch manchmal zu seinem Gott, auch wenn er es jetzt nicht so sagt, ich denke mal schon. (weiblich, 16 Jahre, Experimentalistische Hedonisten, keine Religionsgemeinschaft) 


\section{Außerhalb des eigenen Freundeskreises kommt man am ehesten über die Schule mit dem Thema Religion in Kontakt.}

Auch wenn die Jugendlichen in ihrem eigenen Freundeskreis keine Andersgläubigen haben, kennen sie Jugendliche unterschiedlicher Glaubensgemeinschaften aus der Schule oder haben sich zumindest im Religions- und Ethikunterricht mit den verschiedenen Religionen beschäftigt.

( Religion ist nur in der Schule vorhanden. Es gibt das Fach "Religion“. Und nur da erfährt man vom Glauben der Anderen. Gäbe es dieses Fach nicht, würde ich auch nicht wissen, welche Religion die Anderen haben. Das ist bei uns kein Thema. Niemand bei uns hat Vorurteile. Und niemand sieht sich als Angehöriger einer Religion. Man erfährt das nur über die Schule selber. (männlich, 16 Jahre, Adaptiv-Pragmatische, orthodox)

(7) Mich interessiert das eigentlich gar nicht wirklich, und deswegen muss ich das auch nicht wissen. Also man behandelt es ja dann von der 5. bis zur 9. Klasse in der Schule, und da weiß man ja eigentlich schon darüber was, und deswegen muss ich mich jetzt auch nicht weiterhin damit befassen. (männlich, 15 Jahre, Prekäre, keine Religionsgemeinschaft)

Genauso wie beim eigenen Freundeskreis wird die Möglicheit, Menschen aus anderen Glaubensgemeinschaften in der Schule zu begegnen, durch Stadt-Land- und West-Ost-Unterschiede stark beeinflusst: Auf dem Land und in Ostdeutschland begegnen die Jugendlichen deutlich seltener anderen Religionen. Entsprechend vermischen sich hier oftmals religiöse und kulturelle Andersartigkeit in der Wahrnehmung der Jugendlichen - beispielsweise sind andere Religionen automatisch mit anderen Kulturen verknüpft, d. h. man "hat" eine andere Religion, weil man aus dem Ausland kommt.

(7) Also ein asiatischer Freund von mir, der hat das halt so ein bisschen durch seine Eltern. Der ist nicht gläubig oder so. Der hat halt so Bilder, wie zum Beispiel so die ganze Familie auf einem fliegenden Teppich oder so im Wohnzimmer hängen. (männlich, 17 Jahre, Adaptiv-Pragmatische, keine Religionsgemeinschaft) 


\section{Freundschaften zwischen Jugendlichen mit und ohne Religionszugehörigkeit beruhen auf gegenseitiger Toleranz.}

Jugendliche, die nicht Mitglied in einer Glaubensgemeinschaft sind, haben Freundinnen und Freunde aus verschiedenen Glaubensgemeinschaften oder kennen zumindest entsprechende Jugendliche aus der Schule. Im gemeinsamen Alltag spielen Glaube und Religion meist keine große Rolle. Man erwartet und praktiziert gegenseitige Toleranz sowohl im Hinblick auf die Religionsausübung als auch bei der persönlichen Lebensführung. Man stört sich beispielsweise nicht daran, wenn andere aufgrund ihres Glaubens regelmäßig zur Kirche gehen, beten oder kein Schweinefleisch essen. Umgekehrt erwartet man von gläubigen Jugendlichen bei Themen wie Homosexualität oder Abtreibung, dass sie sich tolerant zeigen und nicht Regeln aus ihrer Religion auf die Allgemeinheit übertragen.

Typische

Zitate zur Illustration
Ein Freund von mir, der ist halt schwarz, aber das ist halt so ein cooler Schwarzer, der ist halt so lässig drauf, und der ist halt christlich und geht auch immer in die Kirche. Sollte man ja auch, glaube ich, wenn man Christ ist. Aber sonst befasse ich mich eigentlich gar nicht damit. (weiblich, 15 Jahre, Prekäre, keine Religionsgemeinschaft)

( Meine ganzen Freunde glauben auch an nichts. Ich habe eine Freundin, die glaubt an Gott, das ist okay für mich. Ich kann damit umgehen, sie geht jeden Sonntag auch zum Gottesdienst, was auch okay ist und das war's. Also ich habe damit auch nicht viel zu tun. (weiblich, 17 Jahre, Materialistische Hedonisten, keine Religionsgemeinschaft)

( Ich habe zwei Freunde, die sind christlich, und ich habe auch einen Kumpel, der jetzt so richtig auf den Buddha fixiert ist. Der sagt, wenn man jeden Tag vom Buddha den Bauch reibt, dass das Glück bringt. Und jeden Morgen, auch auf der Klassenfahrt, da hatte der so einen Mini-Buddha mit, macht der so: Bring mir Glück! Ich finde es auch gut, dass jeder so seines hat, wenn er gläubig ist, mein Gott! Wenn er halt an Gott glaubt, an den Buddha, an ... Ist mir doch egal. Es ist mir egal, 
welchen Glauben er hat. (weiblich, 14 Jahre, Experimentalistische Hedonisten, keine Religionsgemeinschaft)

( Ich kenne viele Freunde, die Moslems sind. Ja, aber da gibt es deswegen auch keine Streitigkeiten oder so. [INT: ABER GIBT ES UNTERSCHIEDE?] Naja ... Bei den Moslems auf jeden Fall, dass die kein Schweinefleisch essen so. Das muss man natürlich so auch akzeptieren und natürlich denen nichts voressen oder bzw. hier so Schwein anbieten, das ist dann absolutes No-Go, sage ich mal. Aber ansonsten kein großer Streit. (männlich, 16 Jahre, Adaptiv-Pragmatische, keine Religionsgemeinschaft)

Interviewerin: weiblich, 16 Jahre, Adaptiv-Pragmatische, muslimisch

WiE FINDEST DU ES, DASS MANCHE ReLIGIONEN UNTERDRÜCKT WERDEN? Also ICH MEINE Z. B., DIE ALEVITEN WERDEN JA MEIST NICHT IM ISLAM ANGENOMMEN, ODER ATHEISTEN ÜBERHAUPT WERDEN JA ALS UNGLÄUBIGE UND WAS WEISS ICH BETITELT. WIE FINDEST DU DAS?

( Das finde ich natürlich nicht gut. Das ist auch nicht schön, was die damals mit den Juden gemacht haben, ist auch nicht korrekt. Darum sollte jeder seine eigene Religion ausleben können und daran glauben können. Du bist heute bei den Aleviten und ich bin bei den Sunniten, und wir sind aber Freunde. Und das ist auch gut so. Und mir ist es egal, an was du glaubst und wie du bist, wie du dich anziehst oder was du machst, ob du betest oder ob du nicht betest, ob du fastest. Zum Beispiel ich faste jetzt bei Ramadan, aber du fastest nicht. Und du fastest aber dafür einen anderen Monat, und das ist auch gut so.

\section{FANDEST DU DAS FASTEN SCHWER?}

(I)h finde das natürlich schwer, gerade in diesen Sommertagen finde ich es ganz schwer. Essen ist ja nicht so schwer, aber trinken. Zum Glück gibt es jetzt noch zwei Tage, aber ich glaube auch daran, wenn ich diesen fast einen Monat Fasten durchgehalten habe, dass ich ein bisschen näher an Gott heran komme, und ich fühle mich dann auch besser. Ja, es hilft mir einfach, aber meine Mutter hat es mir so beigebracht, deine hat es dir so beigebracht, darum ist es ganz ok. 
FREUST DU DICH AUF DAS ZUCKERFEST?

( Ja, ich freu mich, weil erstens wir diesen einen Monat bestanden haben, und zweitens, dass wir dann Geld kriegen. Und ja, wir werden dann alle beisammen sitzen und ... ja, es wird Spaß machen.

\subsection{Umgang mit religiösen Konflikten}

\section{Religiöse Konflikte werden vor allem mit den aktuellen Auseinandersetzungen rund um den Islam assoziiert.}

Die befragten Jugendlichen denken bei religiösen Konflikten an erster Stelle an die gewalttätigen Auseinandersetzungen in Syrien bzw. an den Islamischen Staat. Erwähnt werden aber auch islamistischer Terror in Europa, Al-Qaida, der Konflikt zwischen Israel und den Palästinensern oder die Taliban. Viele äußern ihr Unverständnis in Bezug auf diese Konflikte, und es fällt innen schwer, die Gründe für die Auseinandersetzungen nachzuvollziehen. Entsprechend vermischen sich in ihrer Wahrnehmung religiöse und kulturelle Konflikte, so dass beispielsweise auch der Konflikt zwischen Kurden und Türken in diesem Kontext erwähnt wird.

Typische Zitate zur Illustration
[INT: KenNST Du RELIGIÖSE KONFLIKTE IN DER WeLt?] Ja, den IS zUm Beispiel. Dem geht es ja auch um die Religion (männlich, 16 Jahre, Adaptiv-Pragmatische, orthodox)

(7) [INT: ES GIBT JA VIELE RELIGIÖS BEgRÜNDETE KonfLIKTE UND KRIEGE, BEKOMMST DU DAVON IRGENDETWAS MIT?] Das Einzige, was mir dazu einfällt ist das mit der IS und die Muslime mit ihrer Scharia. Dass die sagen, das ist alles gewollt von Allah und so etwas. Aber davon halte ich gar nichts. Das finde ich total krank. Aber etwas anderes fällt mir jetzt dazu auch nicht ein. (weiblich, 16 Jahre, Experimentalistische Hedonisten, evangelisch)

(7INT: ES GIBT JA VIELE RELIGIÖS BEGRÜNDETE KRIEGE UND KonfLIKTE AUf DeR WeLt. WAS BEKOMMST DU SO DAVON MIt?] Ja, dass die 
Moslems in anderen Ländern direkt als Terroristen bezeichnet werden. [INT: WIE DENKST DU DENN DARÜBER?] Nicht jeder Mensch mit schwarzen Haaren und langem Bart ist Terrorist. (männlich, 17 Jahre, Materialistische Hedonisten, muslimisch)

[INT: ZURZEIT IST JA AUCH ZIEMLICH VIEL LOS MIT RELIGIÖSEN KONFLIKTEN. FÄLLT DIR DA AUCH ETWAS DAZU EIN?] Ja, also gerade Charlie Hebdo, das war auch ein ziemlich zentraler Punkt. Da habe ich ein Gedicht drüber geschrieben. Es heißt „Bleistiftsoldaten“. Gerade, weil ich das Thema eigentlich ganz zentral fand. (weiblich, 16 Jahre, Sozialökologische, katholisch)

( Ja, ich bekomme mit, dass die Israelis die Palästinenser, also an denen Völkermord begehen. [INT: UND WAS NOCH?] Dass die IS auch Menschen umbringt, aber ich habe da nicht so viel Interesse dran. Wie soll ich sagen, es tut jeder jedem Unrecht. Ist so. Es wird jeder Volksgruppe, wenn ich das so sagen darf, Unrecht getan. Deswegen, es wird immer so weiter gehen. Die Menschheit wird es nie schaffen, die Religion als nur eine Religion anzusehen, als eine friedliche Gemeinschaft. Wird es nie schaffen. Deswegen passiert auch so etwas. (weiblich, 16 Jahre, Konservativ-Bürgerliche, muslimisch)

Und es macht mich traurig, dass Menschen ihren Glauben derart umlegen. Der Islam ist ja praktisch die Religion des Friedens. Und diese Leute sind keine Muslime. Das sind Islamisten. Und was ich auch schade finde, ist, dass das die Menschen verwechseln. Sie verfluchen den Islam, obwohl die Islamisten mit dem Islam nichts zu tun haben. (männlich, 15 Jahre, Sozialökologische, christlich)

[INT: ES GIBT JA VIEL RELIGIÖS BEGRÜNDETE KRIEGE UND KONFLIKTE AUF DER WELT. WAS BEKOMMST DU DAVON SO MIT?] Das verstehe ich nicht. Ich finde, sowas ist einfach dämlich. Ich meine, man kann sich ganz normal unterhalten miteinander. Man kann über seine Probleme reden. Für was gibt es Psychologen? Das sind dann die Dinger, wo ich dann denke: "Euch geht es auch nicht mehr ganz gut." (weiblich, 17 Jahre, Konservativ-Bürgerliche, evangelisch)

Problembewusst zeigen sich vor allem die Lebenswelten in mittlerer und höherer Bildungslage, z. B. Konservativ-Bürgerliche, Adaptiv- 
Pragmatische und Sozialökologische. Materialistische Hedonisten und Prekäre sind weniger gut informiert. Bei den Experimentalistischen Hedonisten vermischen sich am stärksten religiöse und kulturelle Themen. Expeditive sind wohl informiert, zeigen aber wenig Interesse, sich mit dem Thema zu beschäftigen.

Auch wenn ihnen manchmal die genauen Begriffe dafür fehlen, versuchen die Jugendlichen meist zwischen dem Islam als Religion, den verschiedenen Interpretationsmöglichkeiten des Korans und einer religiös begründeten Gewaltausübung zu unterscheiden. Die Jugendlichen, die teilweise eine sehr differenzierte Sichtweise auf die Konflikte haben, nehmen wahr, dass diese bis in ihren Alltag hinein wirken, z. B. in Form von Angriffen auf muslimische Jugendliche in der Schule.

(7) [INT: An WELCHE KONFLIKTE DENKST DU DA SO?] IS größtenteils. Davon hört man auch am meisten. Und das ist halt sinnlos. Das ist keine Religion für mich. Also bei uns auf der Schule gab es einen Fall, da wurde auch ein Mädchen mit Kopftuch zusammengeschlagen, weil die Jungs da was mitbekommen haben von wegen, die gehört dem Islam an, die sind total böse und köpfen Menschen. Aber ich denke halt, wie gesagt, dass das nichts mit der Religion an sich zu tun hat, sondern wirklich nur so ein paar fanatische Spinner sind, die das nur nutzen, um zu sagen, wir führen Krieg oder köpfen Menschen. Das ist für mich etwas komplett anderes als Religion. (männlich, 14 Jahre, Adaptiv-Pragmatische, keine Religionsgemeinschaft)

Religiöse Konflikte, die sich auf andere Religionen und Regionen beziehen und die eine geringere mediale Präsenz haben - z. B. zwischen orthodoxen Juden und Muslimen in Israel, zwischen Buddhisten und Muslimen in Myanmar - sind den meisten Befragten kaum bekannt. 


\section{Religiös begründete Gewalt wird von Jugendlichen aller Religionen aufs Schärfste abgelehnt.}

In der Grundhaltung zu religiösen Konflikten gibt es keine Unterschiede zwischen Jugendlichen, die einer Religionsgemeinschaft angehören und denen, die keiner angehören.

Die meisten können generell nicht nachvollziehen, wie eine Religion als Argumentationsgrundlage für einen Krieg herangezogen werden kann. Für sie ist eine Religion zunächst ein Regelwerk, das vor allem darauf ausgerichtet ist, das friedliche Zusammenleben miteinander zu fördern. Daher sei es auch egal, ob man an Gott, Allah, Buddha oder an niemanden glaube. Religion als Erklärung für Krieg und Konflikt ist aus Sicht von manchen Jugendlichen ohnehin nur ein vorgeschobener Grund. Sie vermuten, dass ohne Religion andere Gründe für gewalttätige Konflikte angeführt würden, z. B. Nationalität oder Hautfarbe. Das sehen auch die Jugendlichen so, die nicht gläubig sind. Sie betrachten islamistische Terroristen eher als Personen mit psychischen Problemen denn als religiös motivierte Aktivisten.

[INT: ES GIBT IN MANCHEN LÄNDERN KRIEGE UND KonfLIKTE WEgEN RELIGION, WAS SAGST DU DENN DAZU?] Das finde ich total den Unsinn. Jeder hat ja seinen Glauben. Früher wurden die Heiden immer so weggescheucht, weil die ja keinen Glauben hatten. Das finde ich eigentlich dumm. Wenn die keinen Glauben haben, dann lass sie doch. Ich finde es eigentlich nur dumm, wenn man sich bekriegt, weil der eine nicht an den anderen Gott glaubt. Im Islam glauben die ja an Allah und so etwas halt alles, und der andere glaubt an Gott oder Christi Himmel oder so etwas alles. Da denke ich mir so, mein Gott, du glaubst an Christus und du an Allah, hast du ein Problem damit? Wenn ja, dann geh in die Ecke und heul! Ich finde es einfach nur dumm, wenn man sich bekriegt, weil der eine nicht an das andere glaubt. Das wäre das Gleiche, wenn zwei kleine Mädchen sich bekriegen, weil die eine an Lillifee glaubt und die andere an Medusa zum Beispiel. (weiblich, 14 Jahre, Experimentalistische Hedonisten, keine Religionszugehörigkeit)

Typische

Zitate zur |llustration 
( Ich finde, das ist einfach Schwachsinn. Die sollen die Einen ihren Glauben leben lassen, und die Anderen sollen an das glauben. was sie wollen. Und das kann ja jedem egal sein, was der andere glaubt. [INT: Du FINDEST ES QUATSCH, WENN AUFGRUND DESSEN KRIEG GEFÜHRT WIRD?] Ja. Es kann ja nicht jedem immer dasselbe gefallen. (weiblich, 15 Jahre, Materialistische Hedonisten, evangelisch)

(7) [INT: KenNSt Du RELIGIÖSE KonflikTE IN DER WeLt?] Ich finde das einfach nur dumm. Ich kann es nicht nachvollziehen, wie man sagen kann, man tötet Ungläubige für einen Gott, der angeblich alle Menschen liebt. Das macht einfach keinen Sinn. (weiblich, 17 Jahre, Adaptiv-Pragmatische, evangelisch)

[INT: WAS DENKST DU ÜBER RELIGIÖSE KONFLIKTE, WAS BEKOMMST DU DAVON MIT?] Ich finde, in der Religion geht es auch darum, dass man andere akzeptiert wie sie sind und ihre Meinung akzeptiert. Ich kann mir nicht vorstellen, dass irgendein Gott in irgendeinem Buch sagt, dass man andere Menschen umbringen soll, nur weil das der Glaube sagt. Das glaube ich nicht, weil das würde kein Glaube sagen. Ich finde das sinnlos einfach. Sinnlos verschwendete Energie, die eigentlich in andere Sachen gesteckt werden könnte. (weiblich, 16 Jahre, Experimentalistische Hedonisten, keine Religionsgemeinschaft)

Besonders bestürzt von der religiösen Begründung der Gewalt zeigen sich die Konservativ-Bürgerlichen und Sozialökologischen Jugendlichen, denen vor allem Frieden, Nächstenliebe und gemeinschaftliches Miteinander wichtig sind. Den Materialistischen Hedonisten, Prekären, aber auch Adaptiv-Pragmatischen fällt es hingegen nicht immer so leicht, eine klare Meinung zu äußern, weil sie sich nicht genügend informiert fühlen. Zudem vermeiden sie es oft, sich eindeutig zu positionieren - gerade weil ihnen nicht klar ist, welche Bedeutung Religion für andere haben kann.

Vor allem Jugendliche ohne Religionszugehörigkeit befürchten im Zuge der aktuellen gewalttätigen Konflikte generelle Vorverurteilungen und Stigmatisierung von bestimmten Religionen und deren Angehörigen.

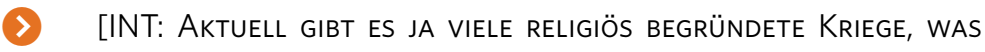
BEKOMMST DU DAVON MIT?] Ich bekomme halt zum Beispiel auch 
hier in Deutschland mit, dass halt viel über Terroristen geredet wird, und dann wird halt so bei so Menschen, die sich halt damit nicht auskennen, gleich gesagt, dass eben alle Muslime Terroristen sind. Dabei muss man die halt schon unterscheiden, weil das ja ganz was anderes ist. Sowas kriege ich halt schon mit, und das finde ich auch total wichtig, eigentlich, weil das ist ja völlig was anderes eigentlich und das kann man ja nicht sagen. (weiblich, 17 Jahre, Materialistische Hedonisten, keine Religionsgemeinschaft)

\section{Muslimische Jugendliche distanzieren sich eindeutig von der Koranauslegung und der Praxis radikaler und gewalttätiger Gruppierungen.}

Auch muslimische Jugendliche äußern sich verständnislos gegenüber radikalen Gruppierungen, die im Namen der Religion agieren. Noch entschiedener als christliche Jugendliche oder Jugendliche ohne Religionszugehörigkeit betonen sie, dass die aktuellen Konflikte nichts mit dem Islam - und damit "ihrer" Religion - zu tun haben. Da die Anhänger des IS für sie unter keinen Umständen als Muslime gelten können, zweifeln sie auch nicht an ihrer Religion selbst, sondern betrachten die Radikalisierungen als abwegige Auslegung des Korans. Rückhalt erfahren sie teilweise durch die eigene muslimische Gemeinde, z. B. indem der Imam ihnen erklärt, dass radikale Islamisten den Islam nicht verstanden hätten.

Sie fühlen sich zwar nicht in die Defensive gedrängt mit Blick auf ihre Religionszugehörigkeit, spüren aber dennoch, dass ihr Glaube in der Gesellschaft falsch wahrgenommen wird, insbesondere vor dem Hintergrund aktueller terroristischer Anschläge. Sie nehmen eine Stigmatisierung gläubiger Muslime durch die christliche Mehrheitsgesellschaft wahr auch durch die mediale Berichterstattung - und wünschen sich, dass der Islam von Andersgläubigen nicht per se negativ beurteilt wird. So wie sie andere ihren Glauben leben lassen, möchten auch sie in ihrer Religionszugehörigkeit ernstgenommen und respektiert werden. 
Typische Zitate zur |llustration

[INT: Findest du TERRORISTEN SIND SCHLECHTE Moslems, WENN Die DAS SO MACHEN?] Also, das sind für mich gar keine Moslems, wenn die den Koran nicht verstehen. Weil im Koran steht was ganz anderes, also dass man sowas nicht machen darf. [INT: UND REDET IHR DA AUCH MANCHMAL DRÜBER IM FREUNDESKREIS SO?] Ja, ab und zu schon. (männlich, 14 Jahre, Materialistische Hedonisten, muslimisch)

(7INT: Was hältst du VON KRIEgen, Die im Namen Von Religion GEFÜHRT WERDEN?] Also ich finde das einfach schwachsinnig, was die da machen. Was die IS einfach macht, finde ich nicht, dass das zum Islam gehört. [INT: OKAY. UND DA SPRICHST DU DANN AUCH DRÜBER? AUCH MIT DEM IMAN?] ICh sage da auch meine Meinung. Und der Iman sagt auch selber, dass das einfach mit der Religion nichts zu tun hat und dass das einfach so eine Gruppe ist, die einfach den Islam falsch verstanden hat. Es gibt immer solche Sonderfälle. (weiblich, 16 Jahre, Materialistische Hedonisten, muslimisch)

(7) Für mich sind das keine Muslime und dass sie unter dem Namen vom Islam Terror machen, das ist keine Bedrohung für mich jetzt zu sagen, ich will kein Moslem mehr sein oder so. Weil für mich sind das keine Muslime. (...) Die Menschen sollten aufhören, über die Religion zu urteilen. Wir sind alle Mensch und Mensch sollten den Mensch beschützen. Aber wir tun genau das Gegenteil. Löwen beschützen auch Löwen gegen Tiger, aber wir sind Feinde gegeneinander. Warum? Ich frage wieder warum, es kommt automatisch immer diese Frage, aber das ist halt so. Ich glaube, die Kriege haben die Menschen auch irgendwie angenommen, zum Lebensbestandteil gemacht, automatisch, weil sie jeden Tag in den Nachrichten etwas hören. (männlich, 15 Jahre, Konservativ-Bürgerliche, muslimisch)

\section{Religiöse Konflikte haben kaum Auswirkungen auf den individuellen Glauben der Jugendlichen.}

Sowohl christliche wie auch muslimische Jugendliche beschäftigen sich intensiv mit den gegenwärtigen auch religiös begründeten Konflikten, sehen aber aus unterschiedlichen Gründen keinen direkten 
Zusammenhang zu ihrer eigenen Religion oder ihrem Glauben. Gerade für muslimische Jugendliche ist die Abgrenzung zwischen religiösen Konflikten und ihrem persönlichen Glauben von wesentlich größerer Bedeutung als für christliche Jugendliche. Aber auch sie sehen ihren eigenen Glauben weitgehend unbeeinflusst von den aktuellen Auseinandersetzungen. Sie haben für sich eine Form des Glaubens gefunden, die sich deutlich von den islamistischen Auslegungen des Korans unterscheidet und umgeben sich mit Gleichgesinnten, um sich gegenseitig in dieser Haltung zu bestärken.

( Ich bin ja auch sehr stark im Glauben. Und ich weiß ja, was richtig und was falsch ist. Und ich lasse mich von solchen Leuten einfach nicht ..., was die so machen. Dann denke ich, okay, ich weiß das schon, dass Typische Zitate zur Illustration das falsch ist. (männlich, 16 Jahre, Materialistische Hedonisten, muslimisch)

(7) [int: Und hat das einen Einfluss auf deinen Glauben? Wenn du HÖRST, ES GIBT EINFACH KRIEGE, DIE SIND DURCH RELIGION BEGRÜNDET?] Naja, Einfluss ... Wie soll ich sagen, also ... Nee, eigentlich nicht. (weiblich, 17 Jahre, Sozialökologische, evangelisch)

(7) [int: Wenn du zUm Beispiel an Kriege denkst, die im Namen ALLAHS OdER GOTTES GEFÜHRT WERDEN, WAS HÄLTST DU DAVON?] Na ja, ich bekomme wenig in den Nachrichten mit, weil ich ja nicht so oft türkische Nachrichten gucke. Aber passiert schon vieles jetzt in der Türkei. [INT: UND WAS DENKST DU DARÜBER?] Nichts Gutes. [INT: ABER WIRKT SICH DAS IRGENDWIE AUF DEINEN GLAUBEN AUS? HAST DU DAS GeFüHL, dAS VERÄNDERT DEN GLAUbEN IRGENDWIE?] Nee. (weiblich, 14 Jahre, Materialistische Hedonisten, muslimisch)

(7INT: Und Wenn Kriege im Namen Von Religionen Geführt WerDEN, WIRKT SICH DAS AUf DEINEN GLAUBEN UND DEINE RELIGION AUS?] Jein, also nein, eigentlich nicht. In dem Sinne, die IS sagen, sie sind Moslems. Aber würde ein Moslem den Koran gelesen haben, also würde dieser Moslem mal den Koran gelesen haben, würde er merken, dass er kein Moslem ist. In jeder Religion, soweit ich weiß, ist es verboten, Menschen zu töten, ihnen die Menschenrechte wegzunehmen. Und im 
Islam ist es genauso wie in allen anderen Religionen. Und ein Gläubiger, in welcher Richtung auch immer, würde Menschen nicht töten. Deswegen sind das einfach nur Psychopathen, die das machen, in jeder Hinsicht, egal von welcher Religion oder welcher Volksgruppe die sind. Die kann man nicht Gläubige nennen, sondern Psychopathen. (weiblich, 16 Jahre, Konservativ-Bürgerliche, muslimisch)

\section{Jenseits religiöser Konflikte betonen Jugendliche die sinnstiftende und orientierende Funktion von Religionen.}

Während Glaube und Religion im Alltag der Jugendlichen eine eher geringe Rolle spielen und sie oftmals nicht genau beschreiben können, welchen Einfluss der Glaube auf sie selbst hat, wird ihnen durch die Frage anch der Rolle der Religionen in den aktuellen Konflikten deutlich, dass diese auch viele positive und wichtige Aspekte haben. Das gilt auch für Jugendliche, die sich keiner Glaubensgemeinschaft zugehörig fühlen. Die Jugendlichen erkennen und beschreiben - eher rational als mit großem emotionalem Involvement - viele Funktionen, die Religionen in Gesellschaften erfüllen, auch wenn sie selbst diese nicht in Anspruch nehmen. Hierzu gehören insbesondere Antworten auf existenzielle Fragen, beispielsweise auf die Frage, was nach dem Tod kommt. Religionen können darüber hinaus auch Orientierungsgeber im Alltag sein und damit Sicherheit und Vertrauen im Leben geben.

( Nein, man braucht Religionen. Man braucht Vertrauen. Die meisten Menschen brauchen Vertrauen darauf, dass nach dem Leben noch etwas kommt. Sonst gäbe es zu viel Angst vorm Sterben. Der Gedanke wäre zu arg, dass man dann nicht mehr da ist. Dass man dann einfach weg ist. Ich glaube, das können die meisten Menschen nicht verstehen. (...) Ich finde, religiöse Leute haben im Prinzip ein sorgenfreieres Leben. Sie müssen sich keine Sorgen machen, was mit ihnen nach dem Tod passiert. Sie sind dann bei ihrem Gott, und dann ist alles gut. Wenn man selber an nichts glaubt, dann geht man einfach. Nein, das hört sich nicht so schön an. (weiblich, 16 Jahre, Experimentalistische Hedonisten, katholisch) 
(1) Wenn der Grund nicht die Religion wäre, gäbe es einen anderen Grund für den Krieg. Ich glaube außerdem, dass Religion wichtig für die Menschen ist. Es ist wichtig für gläubige Menschen, dass sie diesen Glauben haben. Sie haben etwas, das ihnen sagt, wo es langgeht, und wo der Sinn des Lebens ist. Ich selber suche mir das irgendwo anders, weil ich nicht gläubig bin. Aber Glaube bietet schon einen großen Halt. Es gibt eben viele positive Seiten am Glauben. Und die Konflikte sind eben die negative Seite. (weiblich, 15 Jahre, Expeditive, keine Religionsgemeinschaft)

Aus Sicht der Jugendlichen können die Religionen und der persönliche Glauben einen Beitrag für ein gutes Zusammenleben leisten. Sie gehen davon aus, dass alle Religionen im Grundsatz auf ein friedliches Miteinander angelegt sind. Deswegen machen sie sich für Toleranz gegenüber den vielfältigen individuellen Glaubensrichtungen stark.

Die Wahrnehmung von Religionen wird somit nicht allein von den aktuellen Konflikten, die im Namen der Religion geführt werden, bestimmt. Die Jugendlichen haben einen differenzierten Blick auf Religion und ihre Funktion für die Gesellschaft. Vereinzelt wird die Meinung vertreten, dass es in einer Welt ohne Religionen weniger Kriege und soziale Konflikte gäbe, gleichzeitig wird eine "Abschaffung der Religionen" weder als sinnvolle noch machbare Lösung gesehen. Für muslimische Jugendliche ist eine generelle Abschaffung von Religionen überhaupt nicht vorstellbar.

(7) [INT: WAS DENKST du ÜBER SOlCHE RELIGIÖSEN KonflikTE?] Also ich habe da schon eher eine radikale Meinung zu. Und zwar muss ich ganz ehrlich zugeben, wenn es Religion nicht geben würde, dann würde es auch ganz viele Kriege nicht geben. Das ist ja so. Und deswegen halte ich das für relativ unnötig. Und trotzdem muss ich auf der anderen Seite aber auch sagen, werden Religionen missbraucht. Weil eine Religion ist ja eigentlich nicht da, um Kriege zu führen oder dem anderen konsequent eine Meinung aufdrücken zu wollen. Sondern um sich...ich weiß nicht genau warum, aber vielleicht, um zu hoffen, dass nach dem Tod noch etwas kommt, was cool ist und nicht so hoffnungslos durch das Leben zu gehen. Das ist ja auch für viele Leute ein großer Punkt, gläubig

Typische Zitate zur Illustration 
zu sein. Ich finde einfach, man sollte leben und leben lassen. (weiblich, 16 Jahre, Sozialökologische, keine Religionsgemeinschaft)

( Nee, also ich weiß nicht. Ohne meine Religion fühle ich mich nicht ganz. Und deswegen ist es mir schon wichtig, meine Religion zu haben, etwas, woran ich mich binden kann, etwas, wohin ich mich zurückziehen kann. Etwas, wo ich „ich" sein kann, ist mir wichtig. (weiblich, 16 Jahre, Konservativ-Bürgerliche, muslimisch)

(>) Nein. Das würde gar nicht gehen, wenn es keine Religion geben würde. Das wäre komisch. [INT: WAS wüRDE NICHT GEHEN?] Dann würde jeder machen, was er will. Dann würde man gar nicht so darauf achten, was erlaubt ist zum Beispiel. Dann sieht man auch nicht, wer ein guter Mensch ist und wer kein guter Mensch ist, wer Scheiße baut und wer keine Scheiße baut. (männlich, 17 Jahre, Materialistische Hedonisten, muslimisch)

(7INT: WärE ES DANN BESSER, WENN ES GAR KEINE RELIGION GEBEN WÜRDE?] Das kann ich ja nicht entscheiden. Aber das sehen die Leute wahrscheinlich überhaupt nicht so, aber es würde natürlich die Probleme schon lösen. Aber ich denke nicht, dass man das umsetzen könnte oder so. [INT: WAS DENKST DU, WAS SICH DANN HAUPTSÄCHLICH ÄNDERN WÜRDE, WENN MAN DAS KÖNNTE, DASS MAN DIE RELIGION ABSCHAFFT?] Naja, es werden ja viele Probleme dann aus dem Weg geschafft. Mit diesen Kriegen zum Beispiel. Das wäre zum Beispiel schon mal ein Problem weniger. Dann gibt es eben diese Verschleierung zum Beispiel, die manche überhaupt nicht abhaben können. Mir ist das eigentlich auch egal. Sollen sie halt machen. Und auch dieses mit den Frauen in diesen Ländern, dass die total benachteiligt werden und so, das wäre natürlich auch nicht mehr so. (männlich, 17 Jahre, Adaptiv-Pragmatische, keine Religionsgemeinschaft)

\footnotetext{
Open Access Dieses Kapitel wird unter der Creative Commons Namensnennung - Nicht kommerziell 2.5 International Lizenz (http://creativecommons.org/licenses/by-nc/2.5/deed.de) veröffentlicht, welche für nicht kommerzielle Zwecke die Nutzung, Verbreitung und Wiedergabe in jeglichem Medium und Format erlaubt, sofern Sie den/die ursprünglichen Autor(en), den Titel des Werks und die Quelle ordnungsgemäß nennen, einen Link zur Creative Commons Lizenz beifügen und im Falle einer Abwandlung durch einen entsprechenden Hinweis deutlich erkennbar machen, dass Änderungen vorgenommen wurden. Commons Lizenz, sofern sich aus der Abbildungslegende nichts anderes ergibt. Sofern das betreffende Material nicht unter der genannten Creative Commons Lizenz steht und die betreffende Handlung nicht nach gesetzlichen Vorschriften erlaubt ist, ist auch für die oben aufgeführten nicht-kommerziellen Weiterverwendungen des Materials die Einwilligung des jeweiligen Rechteinhabers einzuholen.
} 